\title{
SOHO/CDS observations of waves above the network
}

\author{
P. Gömöry ${ }^{1,2}$, J. Rybák ${ }^{1}$, A. Kučera ${ }^{1}$, W. Curdt ${ }^{3}$, and H. Wöhl ${ }^{4}$ \\ 1 Astronomical Institute of the Slovak Academy of Sciences, 05960 Tatranská Lomnica, Slovakia \\ e-mail: gomory@astro.sk \\ 2 Sterrekundig Instituut, Utrecht University, PO Box 80000, 3508 TA Utrecht, The Netherlands \\ 3 Max-Planck-Institut für Sonnensystemforschung, Max-Planck-Straße 2, 37191 Katlenburg-Lindau, Germany \\ ${ }^{4}$ Kiepenheuer-Institut für Sonnenphysik, Schöneckstr. 6, 79104 Freiburg, Germany
}

Received 6 July 2005 / Accepted 17 November 2005

\section{ABSTRACT}

We analyze temporal variations in the intensities and the Doppler shifts of He I $584.33 \AA$ (chromosphere), O v $629.73 \AA$ (transition region), and Mg IX 368.07 A (corona) measured in and above chromospheric network near disk center with the Coronal Diagnostic Spectrometer (CDS) onboard the Solar and Heliospheric Observatory (SOHO). There is significant correlation between the He I and O v modulations, with O v intensity leading He I intensity by $27.3 \mathrm{~s} \pm 4.6 \mathrm{~s}$ but no significant time shift in the Doppler shift. Cross-correlation between the O V and Mg IX intensities reveals multiple maxima without correlation between their Doppler shifts. Wavelet power analysis gives evidence of intermittent chromospheric and transition-region oscillations with periodicities in the 250-450 s range and of coronal oscillations in the 110-300 s range. Wavelet phase difference analysis shows that the determined time shift between variations of the He I and O v intensities is dominated by waves with about 300 s periodicity. We interpret these results as giving evidence of compressive waves that propagate downward from the transition region to the chromosphere in the particular chromospheric network. We discuss different scenarios regarding origin and source localization of waves, and we speculate on their role in coronal heating above chromospheric network.

Key words. Sun: atmosphere - Sun: oscillations

\section{Introduction}

The proposed mechanisms to heat the solar corona can roughly be divided into two groups: ( $i$ ) dissipation of magneto-acoustic waves excited in the photosphere, and (ii) magnetic reconnection in the corona (see e.g. the review by Narain \& Ulmschneider 1996). We address these two mechanisms here for the quiet sun using ultraviolet spectrometry. Our approach is that the latter may discriminate between the two classes of mechanisms.

The first mechanism, wave heating, is thought to result from the footpoint motions imposed by convective motions on the strong-field fluxtubes that are visible as photospheric bright points concentrated in the magnetic network (Muller et al. 1994). Thus, the photospheric plasma motion continually affects magnetic field lines and excites magneto-acoustic waves. Such waves propagate upward along the magnetic field lines and transfer energy from the photosphere to the upper atmosphere where it may get dissipated after transformation of the waves to smaller spatial scales through phase mixing (Heyvaerts \& Priest 1983) and/or resonant absorption (Ionson 1978; see e.g. the review by Walsh \& Ireland 2003)

The second mechanism, reconnection heating, may occur from outer-atmosphere field topology changes imposed by photospheric footpoint motions (Priest 1982) and/or by field line deformations that produce small-scale reconnection events called nanoflares (Parker 1988) with a duration of a few seconds and scales below the present observational limits. However, a nanoflare should create strong stress in magnetic field lines which may propagate downward as a magnetic body wave. Hansteen et al. (1997) attributed the observed redshift of transition region emission lines to such downward propagating compressive waves. On the other hand, Peter et al. (2004) synthesized coronal line profiles from threedimensional MHD modeling and showed that field-line braiding is a prime candidate for coronal heating in magnetically closed structures.

Determination of the dominant mechanism to heat the outer solar atmosphere in/above chromospheric network is often based on results obtained from analysis of the oscillatory behaviour (Hansteen et al. 2000; Banerjee et al. 2001) or from numerical modeling (Hansteen 1993; Wikstøl et al. 1997). Both techniques suggest the presence of the propagating waves in this part of the solar atmosphere.

In this paper we study wave modulation of ultraviolet emission lines in and above quiet chromospheric network. In particular, we employ cross-correlation and wavelet analysis of the intensities as well as the Doppler shifts of emission lines of $\mathrm{He}$ I (chromosphere), O V (transition region) and Mg IX (coronal) to study waves at different heights and their 

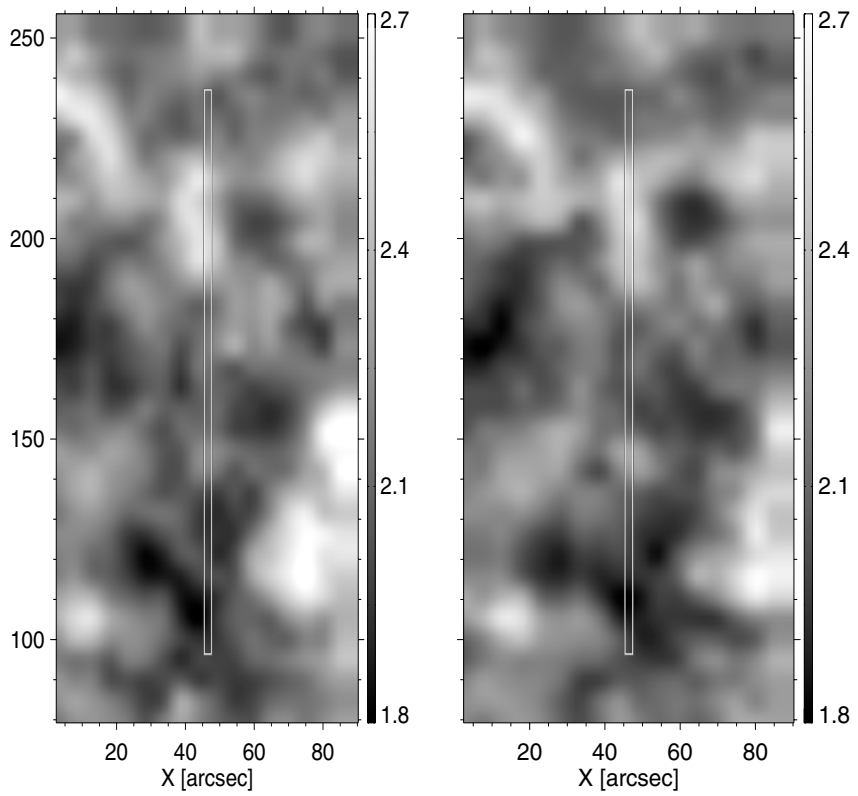

Fig. 1. CDS slit position superimposed on SOHO/EIT images in He II $304 \AA(\log T=4.9)$ at the beginning (left) and end (right) of the CDS measurements. The coordinates of the CDS slit center are $X=46.4 \operatorname{arcsec}, Y=167.1$ arcsec relative to disk center, with $Y$ positive to the north along meridians. Intensity in $\mathrm{W} \mathrm{m}^{-2} \mathrm{sr}^{-1} \AA^{-1}$ is displayed on a logarithmic scale.

direction of propagation. Preliminary results have been reported by Gömöry et al. (2005).

\section{Data and data reduction}

We use data taken with the Coronal Diagnostic Spectrometer (CDS, Harrison et al. 1995) onboard the Solar and Heliospheric Observatory (SOHO, Domingo et al. 1995) in a JOP 78 (Kučera et al. 1999) from 23:25 UT until 23:53 UT on May 14, 1998. A quiet region of the solar atmosphere near disk center (Fig. 1) was observed with the normal incidence part (NIS) of CDS using the 2 arcsec wide and $141 \operatorname{arcsec}$ ( 84 pixels) long slit in three spectral lines, namely: He I $584.33 \AA(\log T=4.5)$, O v $629.73 \AA(\log T=5.3)$ and Mg IX $368.07 \AA(\log T=6.0)$. We should remind here that the He lines have complex formation history which may be influenced by the coronal EUV radiation field (O'Shea et al. 2002). But, very recently Mauas et al. (2005) showed that even in an active region the incident coronal radiation has only very small effect on the UV lines of the He. Therefore we can assume that the detected He I radiation reflects only conditions of the upper chromosphere. A dataset of 190 spectral images with an exposure time of $5 \mathrm{~s}$ and cadence of $9.1 \mathrm{~s}$ was obtained using the "sit-and-stare" observation mode. Due to solar rotation a drift scan of 28.8 min was obtained. The final scanned area is $141 \operatorname{arcsec} \times 4.4$ arcsec. As the spatial extent of chromospheric network features seen in the OV line with CDS is typically about 10 arcsec (Gallagher et al. 1998), variations of spectral line parameters shorter than one hour are likely to be local variations rather then feature drift. Moreover, Fig. 1 demonstrates that the same individual chromospheric network $(Y=185-221$ arcsec $)$ was observed.
Using standard CDS software ${ }^{1}$, the raw measurements were corrected for $\mathrm{CCD}$ readout bias, flat-field, cosmic rays, and other instrumental effects of the CDS/NIS instrument. The measurements were then converted to physical units. For the Mg IX line, binning of 2 pixels along the slit and 5 exposures in time was performed to increase the signal-to-noise. A single Gaussian with a linear background and Poisson statistics (for pixel weighing) were used for fitting of each spectral line profile $^{2}$. Note, that although the Mg IX $368.07 \AA$ has a weak blend of the Mg VII (Brekke et al. 1997), which is $0.6 \AA$ apart, the low signal-to-noise has not allowed to perform a multicomponent fitting of this spectral line. The fitted data product consists of the intensities (i.e. amplitudes of the Gaussian profiles) and the Doppler shifts per spatial pixel per exposure (primary data). The wavelength scales were adjusted using the average redshift/blueshift of the transition region/coronal spectral lines of Peter \& Judge (1999) and laboratory wavelengths of Macpherson \& Jordan (1999). We thus obtained new reference wavelengths of our spectral lines: He I $584.330 \AA$, O V $629.751 \AA, \operatorname{Mg}$ IX $368.064 \AA$. The square root errors of the primary data were also calculated. Only data determined from fits with uncertainty $\chi^{2}$ smaller than $10 \mathrm{erg}^{2} \mathrm{~cm}^{-4} \mathrm{~s}^{-2} \mathrm{sr}^{-2} \AA^{-2}$ were used.

\section{Analysis}

Only data of one part of the chromospheric network, henceforth called quiet network, visible in Fig. 1 on the slit between $Y=207-213 \operatorname{arcsec}$ (i.e. four pixels along the slit) are analyzed here. These data are of the highest signal-to-noise ratio in all spectral lines and they are not influenced by eruptive events. One such event detected in the OvDoppler shift $(Y=187-198$ arcsec) was studied in detail and identified as an explosive event (Gömöry et al. 2003).

The He I, O V and Mg IX intensities and the Doppler shifts were averaged over the four pixels covering quiet network (as specified above) for each exposure. The averaged temporal variations were then smoothed with a running mean of five time steps (Fig. 2).

Cross-correlation functions of the averaged and smoothed temporal variations of the $\mathrm{He} \mathrm{I}, \mathrm{O} \mathrm{V}$ and $\mathrm{Mg}$ IX intensities as well as the Doppler shifts (Fig. 3) were calculated using the algorithm of Gömöry et al. (2004a). In this algorithm the removal of the edge pixel effect and subtraction of background trends are taken into account.

A wavelet power analysis of all temporal variations (Fig. 4) was performed using a technique developed by Torrence \& Compo (1998). The Morlet wavelet (Grossman \& Morlet 1984), as analyzing function, and the value of the wavenumber equal to 6 (high temporal resolution) were used for computation of the continuous wavelet power spectra. Note that wavelet transform suffers from edge effects at both ends of the time series. Therefore, all resulting periods above $500 \mathrm{~s}$ could

\footnotetext{
1 http://solar.bnsc.rl.ac.uk/software/uguide/ uguide.shtml

2 http://orpheus.nascom.nasa.gov/cds/swnote/ cds_swnote_47.ps
} 

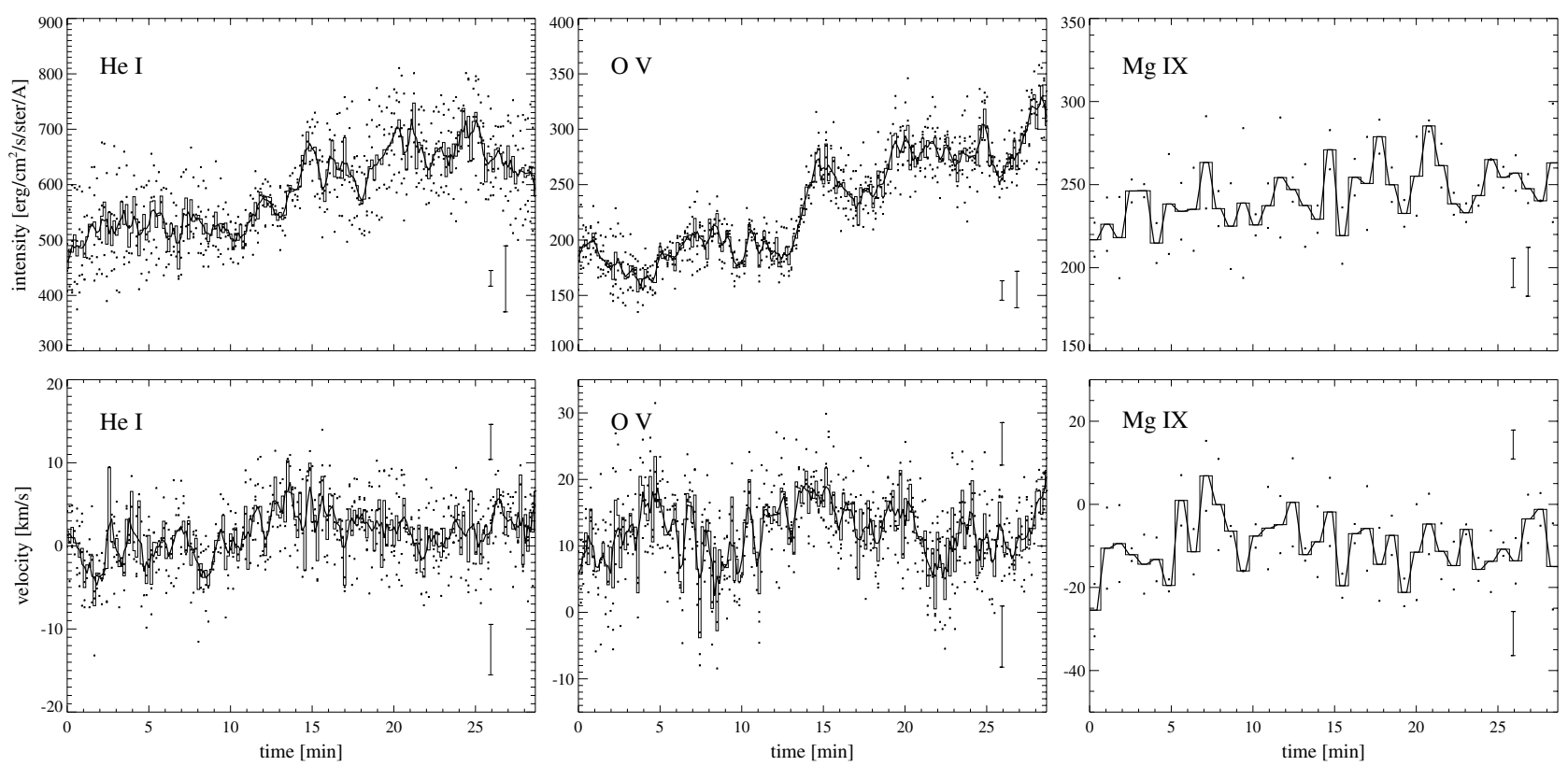

Fig. 2. Temporal variations of the He I, OV and Mg IX intensities (upper row) and the Doppler shifts (lower row). The dots show primary data (four values of four pixels along the slit for each exposure), the histograms display spatially averaged variations and the thick lines show smoothed variations. The typical square root errors of the primary data are $\pm 14.2 \mathrm{erg} \mathrm{cm}{ }^{-2} \mathrm{~s}^{-1} \mathrm{sr}^{-1} \AA^{-1}, \pm 8.8 \mathrm{erg} \mathrm{cm}^{-2} \mathrm{~s}^{-1} \mathrm{sr}^{-1} \AA^{-1}$ and

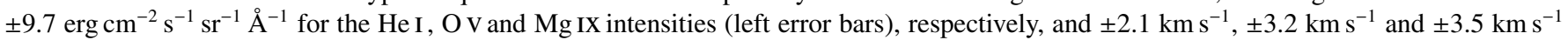
for the $\mathrm{He}$ I, O V and Mg IX Doppler shifts (top error bars), respectively. The mean standard deviations of the primary data from the displayed averaged temporal variations are $\pm 59.6 \mathrm{erg} \mathrm{cm}^{-2} \mathrm{~s}^{-1} \mathrm{sr}^{-1} \AA^{-1}, \pm 16.5 \mathrm{erg} \mathrm{cm}^{-2} \mathrm{~s}^{-1} \mathrm{sr}^{-1} \AA^{-1}$ and $\pm 17.7 \mathrm{erg} \mathrm{cm}^{-2} \mathrm{~s}^{-1} \mathrm{sr}^{-1} \AA^{-1}$ for the He I, O V and Mg IX intensities (right error bars), respectively, and $\pm 3.1 \mathrm{~km} \mathrm{~s}^{-1}, \pm 4.6 \mathrm{~km} \mathrm{~s}^{-1}$ and $\pm 5.8 \mathrm{~km} \mathrm{~s}^{-1}$ for the He I, O V and Mg IX Doppler shifts (bottom error bars), respectively. Positive values of the Doppler shifts correspond to redshift.
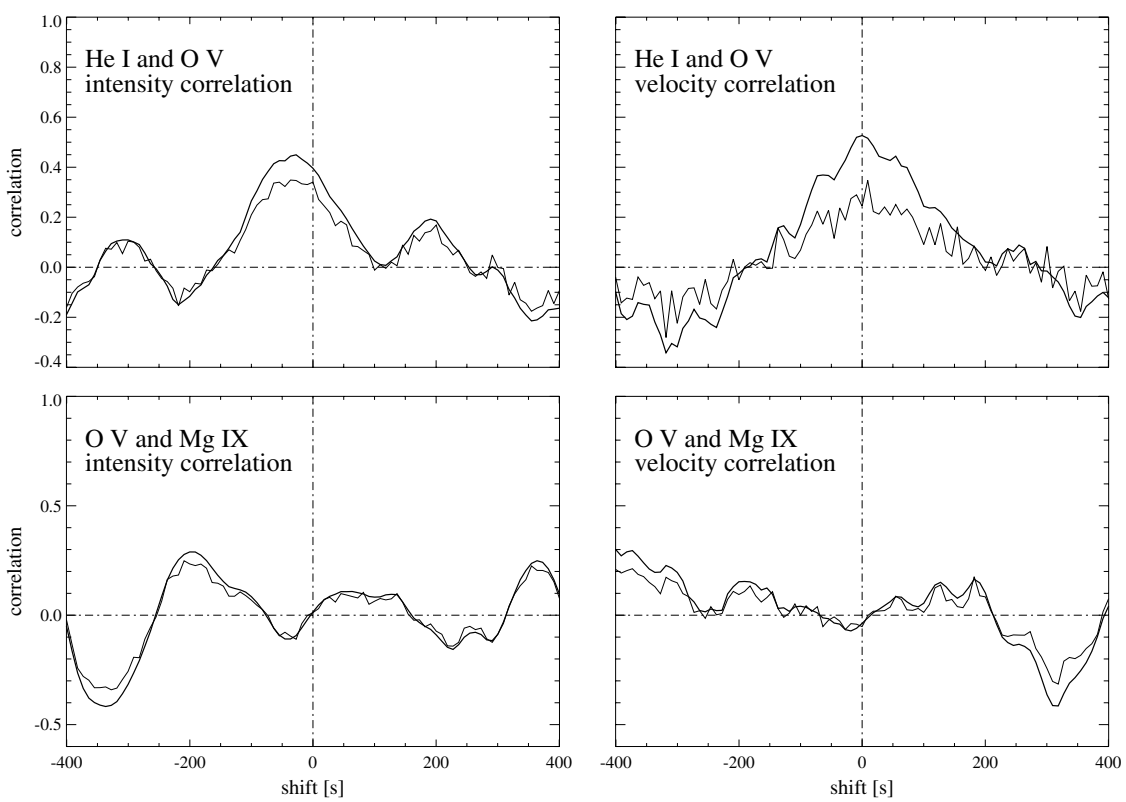

Fig. 3. Cross-correlation functions of the He I and O V intensities and the Doppler shifts (upper row), and of the $\mathrm{OV}$ and Mg IX intensities and the Doppler shifts (lower row). The thin lines show the crosscorrelation functions of the averaged curves and the thick lines show the cross-correlation functions of the smoothed curves. be widely affected and were not taken into account. As significance test, confidence level at $99 \%$ was calculated using a white noise background spectrum.

A wavelet phase difference (Fig. 5) was also calculated following the method of Torrence \& Compo (1998) using the same wavelet and wavenumber as described above. $15 \%$ coherence exceedence level was used as significance test here (Bloomfield et al. 2004).

\section{Results}

\subsection{Chromosphere and transition region}

Figure 2 shows temporal variations of the He I and Ovintensities and the Doppler shifts. The cross-correlations between the He I and $\mathrm{O} v$ intensities and between their Doppler shifts are shown in Fig. 3. Both cross-correlations reach values of about 0.5 . The upper-left panel shows negative 

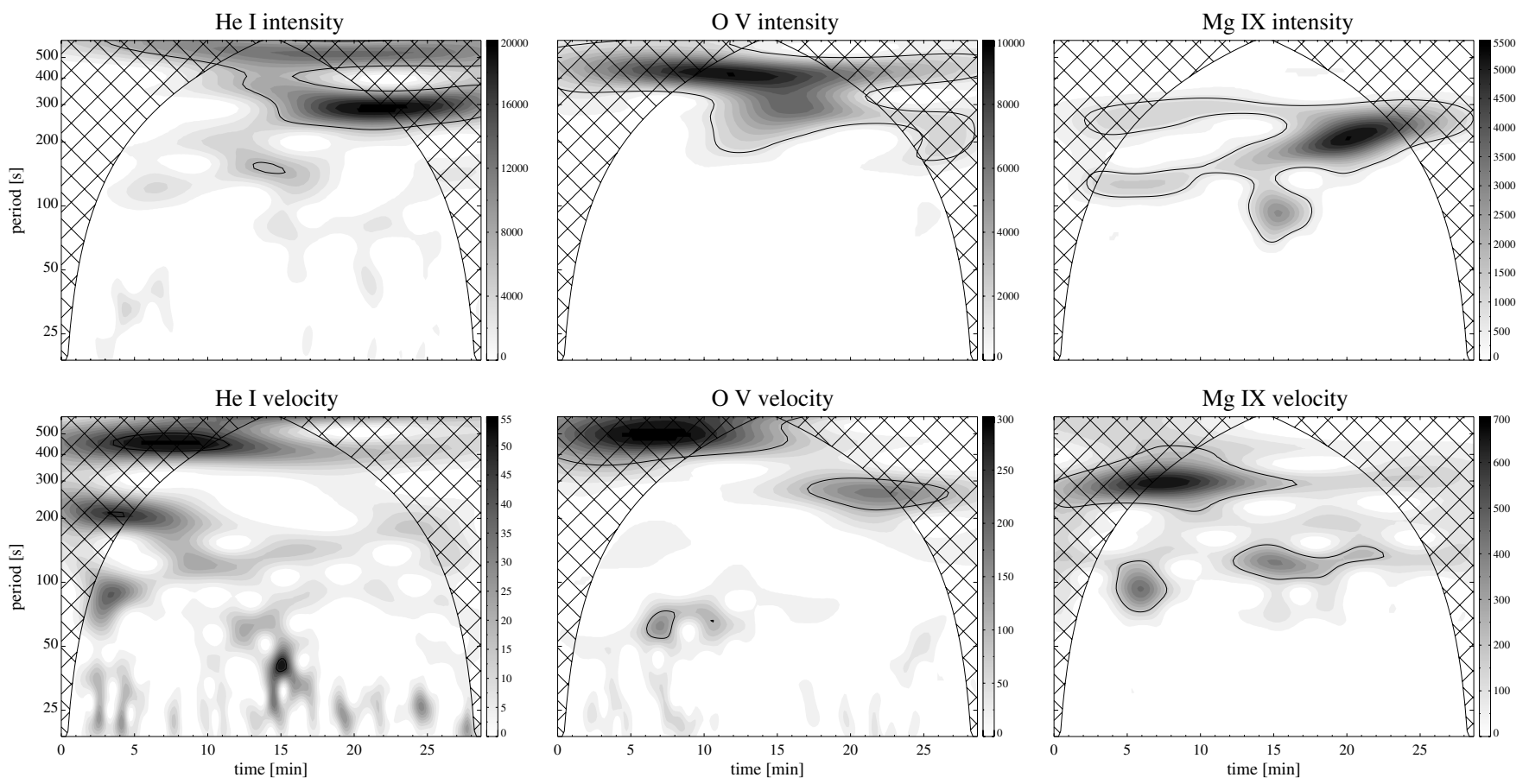

Fig. 4. Wavelet power spectra corresponding to the temporal variations of the He I, O V and Mg IX intensities (upper row) and the Doppler shifts (lower row). The gray scaling shows the power of analyzing wavelets. Contours show locations of the most relevant periods in the time series, i.e. periods with the significance more than $99 \%$. The cross-hatched regions indicate the "cone of influence" (areas where the edge effects become important).

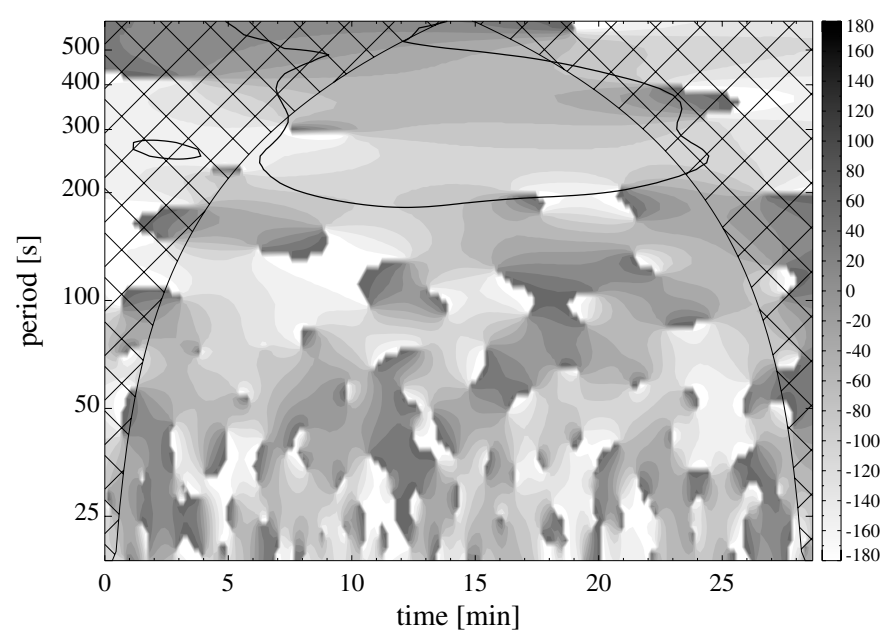

Fig. 5. Wavelet phase difference [degrees] of the He Iand O vintensities. Contoured area corresponds to the $15 \%$ coherence exceedence level, while the cross-hatched area marks the "cone of influence".

time shift of $-27.3 \mathrm{~s}$ between the He I and OVintensities. In contrast, there is no significant time shift between the He I and Ov Doppler shifts. Thus, at least in some temporal subintervals, the changes in the $\mathrm{O}$ V intensity precede changes in the He I intensity without accompanying time shift in the Doppler shifts. Since the temporal resolution of the time series under study is $9.1 \mathrm{~s}$ each inferred time shift is determined with an uncertainty of $\pm 4.55 \mathrm{~s}$.
Figure 4 shows wavelet power spectra from the He I and $\mathrm{O} v$ intensities and from their Doppler shifts. The He I intensity modulation indicates oscillations with periodicity around $300 \mathrm{~s}$, but significantly only in the time interval from $13.3 \mathrm{~min}$ to $28.8 \mathrm{~min}$. The $\mathrm{OV}$ intensity reveals strong power with 250-450 s periodicity. Note that the oscillations with longer periods (around 400s) are relevant during the whole observing sequence whereas oscillations with shorter periods (from $250 \mathrm{~s}$ to $300 \mathrm{~s}$ ) occur only in the time interval from $11.6 \mathrm{~min}$ to $21.6 \mathrm{~min}$. Moreover, no notable power with periods shorter than $200 \mathrm{~s}$ was found for the HeI and Ovintensities. The He I Doppler shift shows only oscillations with lower power which correspond to the periods around $220 \mathrm{~s}$ and around $450 \mathrm{~s}$. These disputable periods are visible during the first $8.3 \mathrm{~min}$ and $15.8 \mathrm{~min}$, respectively. On the other hand, it is interesting that the OvDoppler shift indicates very similar periods with the magnitude around $250 \mathrm{~s}$ and around $450 \mathrm{~s}$ but with considerably higher power. Moreover, longer periods ( $450 \mathrm{~s})$ persists almost in the same time interval as the similar one detected in the He I Doppler shift. Shorter periods ( $250 \mathrm{~s})$ are relevant in the second part of the observing sequence.

As the cross-correlation function of the HeIand $\mathrm{OV}$ intensities reaches a maximum value of around 0.5 only, we used the wavelet phase difference analysis to clarify the significance of the found negative time shift between the $\mathrm{He} \mathrm{I}$ and $\mathrm{O} \mathrm{V}$ intensities (Fig. 5). This analysis reveals that there is relevant negative phase delay of $-20^{\circ}$ which corresponds to the time shift of $-17 \mathrm{~s}$ between the most significant oscillations which occur simultaneously in the temporal variations of the $\mathrm{HeI}$ and $\mathrm{OV}$ intensities, i.e. between oscillations with 
periodicity around $300 \mathrm{~s}$. Wavelet phase difference analysis of the He I and OV intensities shows phase delays also between oscillations with other periods (e.g. phase delay of $-116^{\circ}$ for the oscillations with period around $250 \mathrm{~s}$ ). But, such results were not taken into account as these oscillations were not significant at the same time both in the temporal variations of the $\mathrm{He} \mathrm{I}$ and $\mathrm{O} \mathrm{V}$ intensities.

Slightly different values of the time shift obtained from the wavelet phase difference analysis and from the crosscorrelation of the He I and O V intensities can be caused by the fact that whereas wavelet phase difference analysis provides temporally and frequency resolved time shifts the crosscorrelation gives only averaged time shifts which are obtained as the final results from interference of all different oscillations with their particular time shifts. This also explains lower values of cross-correlation between the $\mathrm{He} \mathrm{I}$ and $\mathrm{O}$ V intensities.

\subsection{Corona}

The temporal variations of the Mg IX intensity and the Doppler shift are displayed in Fig. 2. Note that binning of 2 consecutive pixels along the slit and 5 exposures in time was performed for the $\mathrm{Mg}$ IX line. Both temporal variations were crosscorrelated with the corresponding OV curves (Fig. 3). But, only very ambiguous results were obtained from the crosscorrelation function of the $\mathrm{OV}$ and $\mathrm{Mg}$ IX intensities. As this cross-correlation reaches multiple maximum (for time shifts of $-200 \mathrm{~s}$ and $+360 \mathrm{~s}$ ) it is impossible to determine whether the changes were achieved first in the $\mathrm{O} v$ intensity and then in the Mg IX intensity (negative time shift) or if it was happened reversely (positive time shift). Moreover, no cross-correlation was found between the $\mathrm{O} v$ and $\mathrm{Mg}$ IX Doppler shifts.

The wavelet power spectra from the Mg IX intensity and the Doppler shift were also calculated (Fig. 4). The Mg IX intensity reveals significant power for periodicities in the range from $150 \mathrm{~s}$ to $300 \mathrm{~s}$ with the strongest peak around the period of $230 \mathrm{~s}$. These oscillations are relevant mainly in the second part of the observing sequence, i.e. during the period from $15 \mathrm{~min}$ to $26.6 \mathrm{~min}$. Note that there are also significant oscillations with periodicity around $100 \mathrm{~s}$ but with duration of only $3.3 \mathrm{~min}$. The Mg IX Doppler shift shows relevant power for periods around $300 \mathrm{~s}$ which are significant for the first $15.8 \mathrm{~min}$ of the observations. Oscillations with periodicity around $110 \mathrm{~s}$ are also detected. In this case the bursty nature of the oscillations was confirmed, whereas they were relevant in the time intervals from $4.2 \mathrm{~min}$ to $7.5 \mathrm{~min}$ and from $11.6 \mathrm{~min}$ to $22.5 \mathrm{~min}$.

\section{Discussion}

Several authors have studied the properties of spectral lines observed in the UV and visible part of the electromagnetic wavelength range and reported that the chromospheric plasma localized in network oscillates with a dominant period around 330 s (Kneer \& von Uexküll 1993; Lites et al. 1993; Steffens et al. 1997; Judge et al. 1997; Curdt \& Heinzel 1998; Krijger et al. 2001). Our analysis of the He I intensity revealed very similar dominant oscillations with a periodicity of $300 \mathrm{~s}$ occurring at around $15.5 \mathrm{~min}$. In contrast, Doyle et al. (1998) analyzed intensity modulations of the same chromospheric spectral line (He I $584 \AA$ ) and revealed presence of dominant oscillations with considerable shorter periodicity of $200 \mathrm{~s}$. But we should note that their results were not derived from the most intense regions, so the network nature of those oscillations is uncertain. In case of the He I Doppler shift we found oscillations with periodicities of $220 \mathrm{~s}$ and $450 \mathrm{~s}$ which lifetimes were at least $8.3 \mathrm{~min}$ and $15.8 \mathrm{~min}$, respectively, but they were estimated only with low power. However, Hansteen el al. (2000) revealed that Doppler shifts of chromospheric spectral lines show strong power for periodicities in the range from $160 \mathrm{~s}$ to $250 \mathrm{~s}$ and also relevant oscillations with longer periods around $330 \mathrm{~s}$ and $400 \mathrm{~s}$. Moreover, Banerjee et al. (2001) found that Doppler shift oscillations can extend up to values around $500 \mathrm{~s}$. They also reported the intermittent nature of the chromospheric oscillations with a typical lifetime of $10 \mathrm{~min}$ to $15 \mathrm{~min}$. Note that the discussed periods and typical lifetimes of the Doppler shift oscillations are very similar to our results, thus corroborating our findings.

Temporal variation of the O v intensity and Doppler shift discussed here showed dominant oscillations with the periodicity around $300 \mathrm{~s}$ and around $450 \mathrm{~s}$ for the intensity and oscillations with periods around $250 \mathrm{~s}$ and around $450 \mathrm{~s}$ for the Doppler shift. We also found that the lifetime of the shorter periods was approximately $10 \mathrm{~min}$. Doyle et al. (1998) found that the intensities of the transition region spectral lines show oscillations with dominant periods in the range from $200 \mathrm{~s}$ to $500 \mathrm{~s}$ with the strongest power around $300 \mathrm{~s}$ but moreover they revealed periods with lower relevance approaching $600 \mathrm{~s}$. Hansteen et al. (2000) reported relevant transition region oscillations with periodicities in a band from $100 \mathrm{~s}$ to $330 \mathrm{~s}$ with the strongest peaks around $100 \mathrm{~s}, 200 \mathrm{~s}$ and $330 \mathrm{~s}$ for intensity and $200 \mathrm{~s}$ for Doppler shift. Banerjee et al. (2001) pointed out that intensities of transition region spectral lines show significant power in the range from $250 \mathrm{~s}$ to $500 \mathrm{~s}$ with the strongest peaks around $280 \mathrm{~s}$ and $420 \mathrm{~s}$, whereas the Doppler shifts do not show any significant power within this interval, but oscillate with periods close to $660 \mathrm{~s}$. Moreover, they showed that the lifetime of these oscillations is approximately $20 \mathrm{~min}$ and can extend up to around $40 \mathrm{~min}$ in case of the Doppler shifts. Although we analyzed a rather short data set, the found periods as well as their times of appearance are even though in good coincidence with the previous results. Moreover, our results confirm the presence of two groups of transition region oscillations in the network, i.e. oscillations with shorter periods $(\sim 250 \mathrm{~s})$ as well as with longer periods ( $\sim 400 \mathrm{~s}$ and more).

The periods of the coronal oscillations above chromospheric network are not very well known, although there have been several attempts to determine them. Doyle et al. (1998) reported that the low signal-to-noise detected in the studied coronal line $(\log T=6.0)$ did not allow them to investigate oscillations in the corona. Banerjee et al. (2001) analyzed the same spectral line but although they found some oscillations with the periods around $100 \mathrm{~s}$ and $160 \mathrm{~s}$ the low count rates led to low reliability of these findings. Despite of lower signal-tonoise in the coronal Mg IX line, we found significant coronal oscillations with periods similar to the results of Banerjee et al. (2001). But in addition, we also revealed that these oscillations 

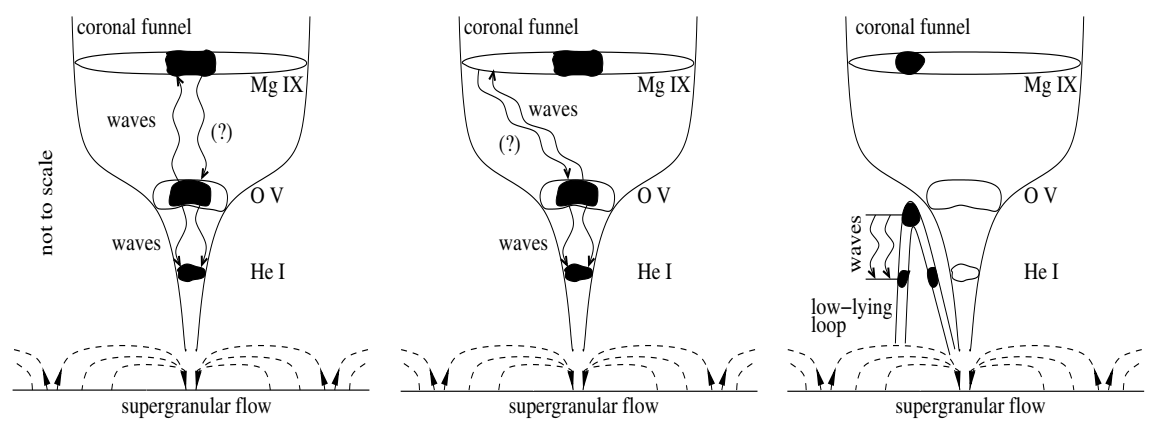

Fig. 6. Topological models of the quiet solar atmosphere above chromospheric network adopted after Gabriel (1976; left and middle) and after Dowdy et al. (1986; right) supplemented by the proposed propagating waves. The black areas correspond to the observed parts of the atmospheric layers while the white contoured areas show the whole layers where the $\mathrm{He} \mathrm{I}$, the $\mathrm{O} V$ and $\mathrm{Mg} \mathrm{IX}$ line radiation can be generated. The wavelike arrows of different orientations describe the propagating waves. could extend up to longer periods ( $200 \mathrm{~s})$. Several authors analyzed the TRACE measurements of the UV emissions from coronal loops and they found coronal oscillations with periods of $300 \mathrm{~s}$ (De Moortel et al. 2002a,b; Marsh et al. 2003). We should note that the oscillations with periods of $100 \mathrm{~s}$ which we found in the Mg IX line intensities were present only during a very short time interval of the observing sequence, so their relevance is very uncertain.

Applying cross-correlation function to the temporal series of the $\mathrm{He} \mathrm{I}$ and $\mathrm{O}$ V intensities we found negative time shift between them, i.e. O V intensity precedes He I intensity. The relevance of this negative time shift was confirmed by wavelet phase difference analysis of these temporal variations which moreover revealed that the obtained time shift is dominated by oscillations with $300 \mathrm{~s}$ periodicity. This demonstrates that the intensity changes start in the transition region and afterwards in the chromosphere. We revealed that the crosscorrelation function of the Doppler shifts of these lines reaches its maximum for the time shift of $0 \mathrm{~s}$. This implies simultaneous macroscopic plasma motion in the chromosphere and transition region. Following these results we can assume that the nonradiative energy had to be transfered from the transition region downward to the chromosphere without any significant bulk mass motion. Assuming a magnetic nature of the chromospheric network (Stenflo 1994; Peter 2001a, 2001b) which creates conditions for the existence of magneto-acoustic waves our results can be explained by downward propagating compressive waves taking place in this part of the solar atmosphere.

Moreover, we found that the mean spectral profiles of these lines, corrected for the instrumental profile, are dominated by non-thermal broadening. While the thermal broadening of the spectral lines is $0.018 \AA$ for the He I and $0.034 \AA$ for the $\mathrm{O}$ V, the non-thermal broadening reaches values of $0.266 \AA$ and $0.275 \AA$, respectively. Instrumental line broadening was estimated by the Gaussian function with the full width at half maximum (FWHM) equal to the slit width (i.e. 2 arcsec). In order to test the reliability of obtained results we also overestimated instrumental line broadening (three times larger value of FWHM) but the resulting non-thermal broadenings remained dominant.

Wikstøl at al. (1997) simulated upward and downward propagating waves in/above chromospheric network and showed that the order of intensity changes detected in spectral lines with different formation temperatures unambiguously determines direction of the wave propagation. They also pointed out that the downward propagating waves affect profiles of the spectral lines much more strongly than upward propagating waves. In particular they showed that the spectral line broadening is much greater if the emitting plasma is influenced by downward propagating waves than by upward propagating waves. Thus, clear observational evidence of the significant non-thermal line broadening can also support the assumption of presence of downward propagating waves.

Curdt et al. (1999) studied quiet solar atmosphere in/above chromospheric network near disk center and cross-correlated the temporal variations of the Ca II K intensities with the intensities of the higher lines of the hydrogen Lyman series and Lyman continuum. They found positive time shifts and interpreted them as evidence of propagating waves that are spreading out from the chromosphere to the transition region. Summarizing their and our results we can assume that both downward as well as upward propagating waves can occur between the quiet chromospheric and transition region layers in the network at different times.

The ambiguous interpretation of the cross-correlation function of the $\mathrm{OV}$ and $\mathrm{Mg}$ IX intensities (multiple maximum) does not allow to determine if the proposed waves originate in the corona and then spread down to lower parts of the solar atmosphere or if they originate in the transition region and later affect the chromosphere and corona (Fig. 6, left). Hansteen (1993) studied perturbations of the coronal magnetic field lines (nanoflares) and reported that pure acoustic waves as well as magneto-acoustic waves excited by nanoflares can effectively propagate downward (i.e. they are not dumped). They also found that slower acoustic waves reach the transition region approximately $30 \mathrm{~s}$ earlier than the chromosphere what is in good agreement with determined time shift between the He I and O V intensities. Calculation of Hansteen et al. (1997) furthermore showed that these waves are hardly detectable in the Mg IX line what can explain why we have not found their clear signature in this spectral line.

Moreover, if we follow Gabriel's model of the transition region (Gabriel 1976) then it is also possible that the observed parts of the transition region and the corona were not magnetically coupled. In this case the waves could be magnetically driven from an unobserved part of the corona to the observed part of the transition region but also vice versa (Fig. 6, middle). This suggestion is supported by TRACE co-observations in the Fe IX $171 \AA$ Å channel (Gömöry et al. 2004b) which show a much more extended area of coronal emission above chromospheric network under study. So the assumption that the waves could originate in the part of the corona unobserved by the CDS instrument is not in contradiction with our findings. 
On the other hand, low cross-correlation of the OV and $\mathrm{Mg}$ IX intensities and no cross-correlation between their Doppler shifts could predict that the signals discussed here originated in different magnetic structures (Fig. 6, right). The multicomponent model of the chromospheric network (Dowdy et al. 1986; Peter 2001a) predicts the existence of small lowlying loops next to the magnetic funnels. Although the radiation emitted from these different structures can be in principle resolved (Peter 2001a,b) the CDS spectral resolution is not high enough to achieve this goal. Following this model it can be alternatively assumed that the observed downward propagating waves occur only in low-lying loops and do not affect the corona above the particular chromospheric network.

\section{Conclusion}

Although we are not able to localize the original source of waves, we clearly show the presence of the downward propagating compressive waves spreading from the transition region to the chromosphere. Moreover, the time shift between the $\mathrm{He} \mathrm{I}$ and $\mathrm{O} \mathrm{V}$ intensities discussed here corresponds with the results determined by Hansteen (1993) for the downward propagating compressive waves excited by nanoflares in corona. This probably points to the coronal origin of the proposed waves. Following this we can state that our observational findings do not conflict with predictions derived for the reconnection heating mechanism of the outer solar atmosphere above chromospheric network.

Acknowledgements. CDS is part of SOHO, the Solar and Heliospheric Observatory, which is a mission of international cooperation between ESA and NASA. P.G., J.R. and A.K. are grateful to the Slovak grant agency VEGA for support of this work (grant No. 2/6195/6). This research is part of the European Solar Magnetism Network (EC/RTN contract HPRN-CT-2002-00313) and part of the Marie Curie Fellowship Stay at the NOVA training site (contract HPMT-CT2001-00245). P.G. is grateful to Prof. R. J. Rutten for his comments and corrections. Wavelet software was provided by C. Torrence and G. Compo (http://paos . colorado. edu/research/wavelets/).

\section{References}

Banerjee, D., O’Shea, E., Doyle, J. G., \& Goossens, M. 2001, A\&A, 371,1137

Bloomfield, D. S., McAteer, R. T. J., Lites, B. W., et al. 2004, ApJ, 617,623

Brekke, P., Kjeldseth-Moe, O., Brynildsen, N., et al. 1997, Sol. Phys., 170,163

Curdt, W., \& Heinzel, P. 1998, ApJ, 503, L95

Curdt, W., Heinzel, P., Schmidt, W., et al. 1999, in Magnetic Fields and Solar Processes, ed. A. Wilson, The 9th European Meeting on Solar Physics, September 12-18, 1999, Florence, Italy, ESA SP-448, 177

De Moortel, I., Hood, A. W., Ireland, J., \& Walsh, R. W. 2002a, in Solar Variability: From Core to Outer Frontiers, ed. A. Wilson, The 10th European Solar Physics Meeting, September 9-14, 2002, Prague, Czech Republic, ESA SP-506, 509

De Moortel, I., Ireland, J., Hood, A. W., \& Walsh, R. W. 2002b, A\&A, 387, L13

Domingo, V., Fleck, B., \& Poland, A. I. 1995, Sol. Phys., 162, 1
Dowdy, J. F., Rabin, D., \& Moore, R. L. 1986, Sol. Phys., 105, 35

Doyle, J. G., van den Oord, G. H. J., O’Shea, E., \& Banerjee, D. 1998, Sol. Phys., 181, 51

Gabriel, A. H. 1976, Phil. Trans. Roy. Soc. London, A281, 339

Gallagher, P. T., Phillips, K. J. H., Harra-Murnion, L. K., \& Keenan, F. P. $1998, A \& A, 335,733$

Gömöry, P., Rybák, J., Kučera, A., Curdt, W., \& Wöhl, H. 2003, Hvar Obs. Bull., 27, 67

Gömöry, P., Rybák, J., Kučera, A., Curdt, W., \& Wöhl, H. 2004a, in Coronal Heating, ed. R. W. Walsh, J. Ireland, D. Danesy, \& B. Fleck, SOHO 15 Conference, September 6-9, 2004, St. Andrews, Scotland, UK, ESA SP-575, 400

Gömöry, P., Rybák, J., Kučera, A., Curdt, W., \& Wöhl, H. 2004b, in Waves, Oscillations and Small-scale Transient Events in the Solar Atmosphere: A Joint View from SOHO and TRACE, ed. H. Lacoste, SOHO 13 Conference, September 29-October 3, 2003, Palma de Mallorca, Balearic Islands, Spain, ESA SP-547, 303

Gömöry, P., Rybák, J., Kučera, A., Curdt, W., \& Wöhl, H. 2005, Hvar Obs. Bull., 29, 71

Grossman, A., \& Morlet, J. 1984, SIAM J. Math, 15, 723

Hansteen, V. H. 1993, ApJ, 402, 741

Hansteen, V. H., Maltby, P., \& Malagoli, A. 1997, in Magnetic Reconnection in the Solar Atmosphere, ed. R. D. Bentley, \& J. T. Mariska, Yohkoh Conference, March 20-22, 1996, Bath, ASP Conf. Ser., 111, 116

Hansteen, V. H., Betta, R., \& Carlsson, M. 2000, A\&A, 360, 742

Harrison, R. A., Sawyer, E. C., Carter, M. K., et al. 1995, Sol. Phys., 162,233

Heyvaerts, J., \& Priest, E. R. 1983, A\&A, 117, 220

Ionson, J. A. 1978, ApJ, 226, 650

Judge, P. G., Carlsson, M., \& Wilhelm., K. 1997, ApJ, 490, L195

Kneer, F., \& von Uexküll, M. 1993, A\&A, 274, 584

Krijger, J. M., Rutten, R. J., Lites, B. W., et al. 2001, A\&A, 379, 1052

Kučera, A., Curdt, W., Fludra, A., Rybák, J., \& Wöhl, H. 1999, in JOSO Annual Report 1998, ed. A. Antalová, H. Balthasar, \& A. Kučera, Astronomical Institute, Slovak Academy of Sciences, Tatranská Lomnica, 149

Lites, B. W., Rutten, R. J., \& Kalkofen, W. 1993, ApJ, 414, 345

Macpherson, K. P., \& Jordan, C. 1999, MNRAS, 308, 510

Marsh, M. S., Walsh, R. W., De Moortel, I., \& Ireland, J. 2003, A\&A, 404, L37

Mauas, P. J. D., Andretta, V., Falchi, A., et al. 2005, ApJ, 619, 604

Muller, R., Roudier, Th., Vigneau, J., \& Auffret, H. 1994, A\&A, 283, 232

Narain, U., \& Ulmschneider, P. 1996, Space Sci. Rev., 75, 453

O'Shea, E., Muglach, K., \& Fleck, B. 2002, A\&A, 387, 642

Parker, E. N. 1988, ApJ, 330, 474

Peter, H., \& Judge, P. G. 1999, ApJ, 522, 1148

Peter, H. 2001a, A\&A, 374, 1108

Peter, H. 2001b, in Solar Encounter, ed. B. Battrick, \& H. Sawaya-Lacoste, First Solar Orbiter Workshop, May 14-18, 2001, Puerto de la Cruz, Tenerife, Spain, ESA SP-493, 327

Peter, H., Gudiksen, B. V., \& Nordlund, A. 2004, ApJ, 617, L85

Priest, E. R. 1982, Solar Magnetohydrodynamics (Dordrecht, Holland: D. Reidel Publishing Company)

Steffens, S., Deubner, F. L., Fleck, B., et al. 1997, in Advances in Physics of Sunspots, ed. B. Schmieder, J. C. del Toro Iniesta, \& M. Vázquez, 1st Advances in Solar Physics Euroconference, ASP Conf. Ser., 118, 284

Stenflo, J. O. 1994, Solar Magnetic Fields: Polarized Radiation Diagnostics (Dordrecht, Holland: Kluwer Academic Publishers) Torrence, C., \& Compo, G. P. 1998, Bull. Amer. Meteor. Soc., 79, 61 Walsh, R. W., \& Ireland, J. 2003, A\&AR, 12, 1

Wikstøl, Ø., Judge, P. G., \& Hansteen, V. H. 1997, ApJ, 483, 972 